\title{
Juxtaposing Mobile Webcasting and Ambient Video for Home Décor
}

\author{
Mudassar Ahmad Mughal \\ Mobile Life @ Stockholm University \\ Box 1263, SE-16429 Kista-Sweden \\ mamughal@dsv.su.se
}

\author{
Jinyi Wang \\ Mobile Life @ Stockholm University \\ Box 1263, SE-16429 Kista-Sweden \\ jinyi@mobilelifecentre.org
}

\author{
Oskar Juhlin \\ Mobile Life @ Stockholm University \\ Box 1263, SE-16429 Kista-Sweden \\ oskarj@dsv.su.se
}

\begin{abstract}
In order to invent and investigate new approaches for the use of enjoying live video, we suggest a combination of emerging mobile webcasting with artistic ambient video, which would enable a form of user generated broadcasts from individually selected cherished places for home decoration. Drawing on the approach of Research through Design we present a study of people who have occasional access to highly appreciated geographical locations, a design instantiation and prototype called LiveNature, as well as a system implementation. We present the result of a technical evaluation, which was conducted during two weeks of deployment. It shows that mobile webcasting provide continuous and stable streams of such a quality that it can be presented for home decoration, and that the video can be combined with real time sensor data to generate aesthetically interesting hybrid media. We also learned that the use of mobile webcasting for home decoration raises new challenges in order to provide unobtrusive and glance based interaction.
\end{abstract}

\section{Categories and Subject Descriptors}

H.5.m [Information interfaces and presentation (e.g. HCI)]: Miscellaneous.

\section{General Terms}

Design, Human Factors.

\section{Keywords}

Ambient video, mobile webcasting, home decoration, liveness, research through design.

\section{INTRODUCTION}

In this paper we investigate the motivations, design and implementation of a new type of multimedia application enabling a new kind of home decoration. In doing so we combine recent advances in mobile webcasting [20], with ambient video format $[5,6,7]$.

The ubiquity of camera phones and consumer-level digital cameras have brought a great anticipation in the public dis- course of how new video services and new forms of use would emerge and become everyday practice among non-professionals [35]. For example news coverage has incorporated high profile examples of

Permission to make digital or hard copies of all or part of this work for personal or classroom use is granted without fee provided that copies are not made or distributed for profit or commercial advantage and that copies bear this notice and the full citation on the first page. Copyrights for components of this work owned by others than ACM must be honored. Abstracting with credit is permitted. To copy otherwise, or republish, to post on servers or to redistribute to lists, requires prior specific permission and/or a fee. Request permissions from Permissions@acm.org.

MUM '14, November 25 - 28 2014, Melbourne, VIC, Australia

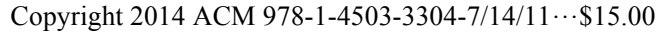

http://dx.doi.org/10.1145/2677972.2677988 videos captured by bystanders to illustrate breaking news reports from events around the world [1]. However, the idea that wide groups of amateurs could adopt mobile webcasting as a form of leisure, while attaining to some form of "production-oriented" standard, i.e. mapped on current professional TV and video formats, has shown to be far from trivial. Previous research has identified a number of general characteristics that make video production challenging, which also hampers its use. The need for editing material before presenting it is a critical burden $[23,25,36]$. Finding relevant content and capturing it in the moment is problematic [20,25]. The media also raises privacy concerns of video capture "anytime and anywhere" [17,25]. Visual storytelling in video is challenging and involves skills that until recently were exclusive to the professional domain. With live mobile webcasting, these problems are emphasized further, as production decisions become increasingly time critical [20,21]. A more mundane type of video format has also emerged in parallel to the professional standard [25], where people model their mobile video capture on snapshot photography, which address the challenges above, but also lose some of the affordances of the new media. In all, the use of mobile webcasting is in its infancy and new ways of using the media will emerge.

In this paper we invent and investigate a third and complementary approach on how mobile webcasting could be modeled. We suggest that it could be used to generate a variety of ambient video [6], which is defined as a form of art presented on highresolution screens [4,7] showing continuous, slow and aesthetically interesting content such as the changes occurring in sceneries of nature [7], which is only viewed casually. The paper is built on the premise that live video becomes abundant and cheap making the combination of mobile webcasting and ambient video possible, since a blend might address some of the challenges described above. First, producing video is time consuming and sometimes interferes with other practices at hand. Live ambient video requires only initial attention when placing cameras, and will then provide continues meaningful broadcast over a long time. Second, early users of mobile webcasting struggled with understanding what would be of interest to the audience as well as how to tell a story with video [20]. The ambient format provide guidelines as to what to record, and make the selection easier since the prolonged broadcast make it possible for a producer to be the same as the viewer.

Furthermore, the suggested combination brings new opportunities to the area of ambient video. It adds mobility and low cost in capture, which make it possible for the many to produce content. Live broadcast is also a constant familiar and popular part of television and immediacy of this media has a "magical" component, which illuminates its cultural impact [20,32]. Mobile broadcasting applications differ e.g. from earlier webcam technologies in that the cameras are wireless, which enables 
recording content from anywhere within the reach of a mobile network, and extended physical control of the device for doing camerawork. Users can therefore select to broadcasts sceneries that are of individual interest e.g. from a cherished place. The availability of other real-time sensor data makes it possible to generate hybrid media i.e. "the simultaneous appearance of multiple media within the same frame" [26]. Hybrid media is already becoming the norm in the moving-image culture since 1990s, with cinematography, graphics, photography, animation and typography being combined in numerous ways. With the advances of sensor technology, such opportunities emerge also in mobile webcasting. Finally, cheap mobile technology can be used to provide ambient interfaces not only for art galleries, but also as home decoration $[2,28,29]$.

We take a Research through Design (RtD) approach where knowledge and decision are embedded in the design of an artifact [42]. RtD is commonly used to invent and investigate in novel and complex settings. By generating a concrete design instance, we acquire an exemplar that indicates the potential of combining technologies and users' interest, to realize a novel concept. It includes making explicit theoretical influences and design considerations, and in this case providing early technical feedback and lessons learned.

Our key contribution can be summarized as follows. We provide a small ethnographic field study among people who have a cherished place i.e. a place they value highly, which is different from where they live. It shows how and when they visualize such place in their mind's eye, when they are not present. We present a design concept, which has been generated through a number of design exercises in dialogue with stakeholders of a large furniture manufacturer. We also present an implementation of the design concept, called LiveNature, which combines mobile webcasting and ambient video for home decoration, and introduce new features to allow easy installation at cherished places. An output system has been installed in an apartment in the south of Sweden, as part of a large furniture manufacturer's test of future concepts. Finally, we provide a technical evaluation and lessons learned from a two weeks deployment. It shows that current mobile webcasting technology is an interesting and plausible candidate for live ambient video. LiveNature generated two weeks of continuous multiple broadcasts of compressed video, with a spatial resolution of $640 * 480$, and an average frame rate of 14.6 frames per second. However, the design and implementation of this media as home decoration was more challenging. The requirement of the furniture manufacturer's stake holders led to unconceived technical problems, such as unsuccessful charging given the need to conceal adapters as well as demands on lowered noise levels.

\section{RELATED WORK}

Our work is influenced by two fields of research, i.e. works on ambient video and research done on aesthetics in home.

\subsection{Ambient video}

Bizzocchi [6] describes ambient video as an emerging form of art, which is made possible by the advent of large high definition displays. Its prime characteristics are to be "pleasant", visually interesting and capable of supporting occasional close viewing. Ambient video should change slowly during a given time interval and preferred content include views of natural sceneries e.g. fire or clouds in the sky, since such elements usually involve slow and gradual changes that allow longer and closer examination. He argues that ambient video is an "inter-media" [18] form of art that exists within the shared border zone of video, cinema, photography and painting [7]. Ambient video attracts attention only casually and it demands minimal attention, although it renew its engagement at whatever moment the viewer choose to return to it. It also sustains visual pleasure over a number of repeated viewings. It adapts the conventions of moving images and incorporates it in the reception model of art.

From an art perspective, Bizzochi extends on Eno's idea of ambient works should be both interesting and ignorable at the same time [9], and sustain over a great number of repeated viewings $[5,4]$. In research, there are many systems and artifacts designed with the understanding of ambience as an "unobtrusive" remote connection between people and/or places. Gaver [12] designed awareness systems with the sense of touch and smell to create connection between distant people. Anind et al [3] designed "connected" household objects to reflect activity of remote family members. Live video is used to create ambient connection when Skype session continuously opens a "presence window" [19]. Wadley et al [37] argue that mobile devices are candidate ambient displays that might convey presence, since they are typically always on and often in peripheral vision

Other research works focus on the aesthetical aspects of ambient video, and recognize its value in the home environment. For example, Wang et al [38] propose solution for presenting archived home videos in an ambient way on a high-resolution display in living spaces. Their system automatically selects, stylizes and transitions between videos in a semantically meaningful sequence. In [40] Yussef et al. discuss the possibilities to present complex visual narrative constructions and ambient video paintings given emerging high definition (HD) display technology and other video. They also discuss the need for new post-production techniques and tools to fully realize the aesthetic properties of this new medium.

In all, the concept of ambient video has attracted attention bot in research and in art with the advent of high-resolution screens. But it has not yet been extended to include mobile live streaming in combination with complementary real time sensor data.

\subsection{Home decoration}

A home often accommodates a range of objects. Beyond the instrumental values, these objects are often acquired for their decorative effects to embellish the home interior [2]. And home décor can be understood as a means of constructing both personal and collective identity. Objects can also create a sentimental world of recall, mementoes and souvenirs constructing the dream world of the bourgeois [2]. Research within modern anthropology also inform us the objects in the homes often carry values such as memories, experiences and style beyond utilitarian values [P179 8]. According to Miller, the objects in the domestic space help to convey a deeper relationship between people and their environment [29]. People set up their home environment that represents a "whole", which Miller refers to as an "aesthetic" [30]. "An aesthetic experience...involves a realization of meaning through interaction with the inherent qualities of the object." [8].

As technology entering our homes, it is criticized that current representations of the home tend to emphasize utilitarian versions of work, entertainment, and consumer activity and other values in the home are poorly served by current technologies [16]. Kirk and Sellen have noted that the physical and digital objects in the homes have different kinds of affordances [22]. Digital objects are often hidden away and have no real presence in the home if they 
are lack of physicality. Thus the design of the digital technology needs to take into consideration of the physical objects, whose careful placement and arrangement help to create the visual landscape of the home interior [34].

While researchers articulate the aesthetics and its role and meanings in people's domestic lives, there is also an emergency of digital design for the homes with the focus on aesthetics. A growing body of work tries to explore how digital technology opens new design space for the aesthetics in the homes. Gaver et al. have designed an electronic coffee table that displays slowly moving aerial photography and argue for the design of ludic engagement motivated by aesthetic rather than utilitarian values [15]. Gaver et al. use electroluminescent material to form an interactive tablecloth, what they refer to as an "aesthetic object", to enhance everyday domestic activities [16]. By refining a recognition technology, Meese et al. explore the design of aesthetic decorative patterns for ceramics tableware that enhances the dining experiences [28]. Built on web-based technology, Ylirisku et al. designed a set of web-connected physical artefacts for the "aesthetics" of the home [39]. We extend the concept of home decoration with liveness and peripheral interaction, and suggest new design space for aesthetic explorations.

\section{METHODOLOGICAL APPROACH}

Our design investigation intends to indicate the potential of combining mobile webcasting with ambient videos for designing aesthetic interactions in the homes. Hence, we choose to adopt the $\mathrm{RtD}$ approach, as our attempt is to explore a design space rather than offer a solution to an existing problem. RtD [11] was introduced to the field of Human-Computer-Interaction by Zimmerman et al [42]. It emphasizes the designed artefact as an end product in which the knowledge generated is embedded. RtD has become a widely adopted approach because it embraces the practice-based nature of design investigations [13, 39]. The knowledge intends to be "generative and suggestive" rather than provide generalization through falsification [14].

Our RtD process started with three field studies of people's love for particular cherished places. The learning from the fieldwork, together with the study of the technology context of live video and theories of aesthetic interactions in homes, are the major sources for the system design explorations. After a series of design experimentation from brainstorming, sketching, building lofidelity prototypes and evaluating them through pilot studies, a resulting concept is further developed to a prototype system. We deployed the prototype in the context of a "Living Lab" by collaboration with an international furniture manufacture company. The Living Lab is an ordinary apartment located in a residential building in the city of Malmö in Sweden. Its interior is designed and furnished by the designers in the company with a strong brand identity. There are a few of novel prototypes installed in the apartment to explore concepts in the domestic environments. The LiveNature system is one of the prototypes. This paper reports the design, implementation and technical evaluation of the LiveNature system in this context. Apart from the three researchers, a designer from the company was also involved in the implementation process and acted as a stakeholder of both the company and future dwellers.

\section{DESIGNING LIVENATURE}

In the following section, we will describe the RtD process in details. We firstly report the learning from the fieldwork and its implications for design. Then we describe the design work, resulting concept, and the technical challenges. Finally we describe the system implementation.

\subsection{Cherished places in the mind's eye}

The design of the LiveNature system is influenced by an idea of people longing for a "cherished place", to which they have access, i.e. a geographical place with which they have a strong emotional attachment. A cherished pace is separate from where they live although revisited on a regular basis. Our idea is that the geographical distance between such a person and the beloved location is an interesting starting point for design research. Preceding the design exercise we did a set of field visits in Sweden to interview people who might be representative of such. We visited a small island in Stockholm archipelago called Bastuholmen, an island in Luleå archipelago called Småskär and a ski resort called Åre.

We did short interview with around 20 persons in the location of their respective cherished places. The interviews had open-ended questions and concerned their relation to the islands and the ski resort, as well as how they imagined it when not there. Since our motivation for doing the interviews was to get inspiration for design, the results have been presented to the rest of the team either as selective transcripts or as transcripts in combination with photos. The results are of two kinds. On the most general level, the interviewees supported our idea of them having a cherished place. A woman on the island of Småskär, who repeatedly returned to this location although living in the city stated: "This is paradise. At least it is my paradise". Other interviewees equally stated that they longed to this place when they were not there and enjoyed it when being there. We also did a study of the design and decoration of private jetties at Bastuholmen, which indicated that this sort of construction was not only practical for parking boats, but often was a beloved family item. On a more detailed level, which was more inspirational to us, several of them conceived of the cherished place in their mind's eye when they were not present. The responses considered what they saw as well as when they saw it. It appeared in their mind's eye when they were longing for it, when they needed to relax, as well as for practical reasons. First a number of people visualized the cherished place when they were longing for it, such as a woman at Småskär who said:

"I'm longing here. I see in my mind's eye when you wake up that you step right out. Walk straight out. The light. Then you make a cup of coffee and drink it outside. I walk around the corner and look towards Finnskär and towards the sea... and I know that my husband normally are out putting in the fishing nets around half six."

Another woman visualized the small houses they were living in at the cherished place, whereas her husband saw: "It's in the canoe somewhere out there (on the sea, our comment) when the weather is very nice". Another woman we interviewed at the ski resort said that she was thinking about it occasionally when she was not there and the she was visualizing: "I see clean, fresh and fresh air". Second, bringing forth the view of the location was done in order to relax. A woman working as a Director had a photo of the houses on Småskär as her desktop background, and explicitly focused on looking at it to decrease her stress. A man at Småskär had previously worked as a manager. When he worked as a manager, and then he had been trained to use a method to relax which included a travel in his mind's eye to a peaceful location. He visualized: “...this bay. I see it in front of me. I am sitting at the terrace overlooking the bay. That is what I see in front of me. This one. Sitting here in the morning eating my breakfast and 


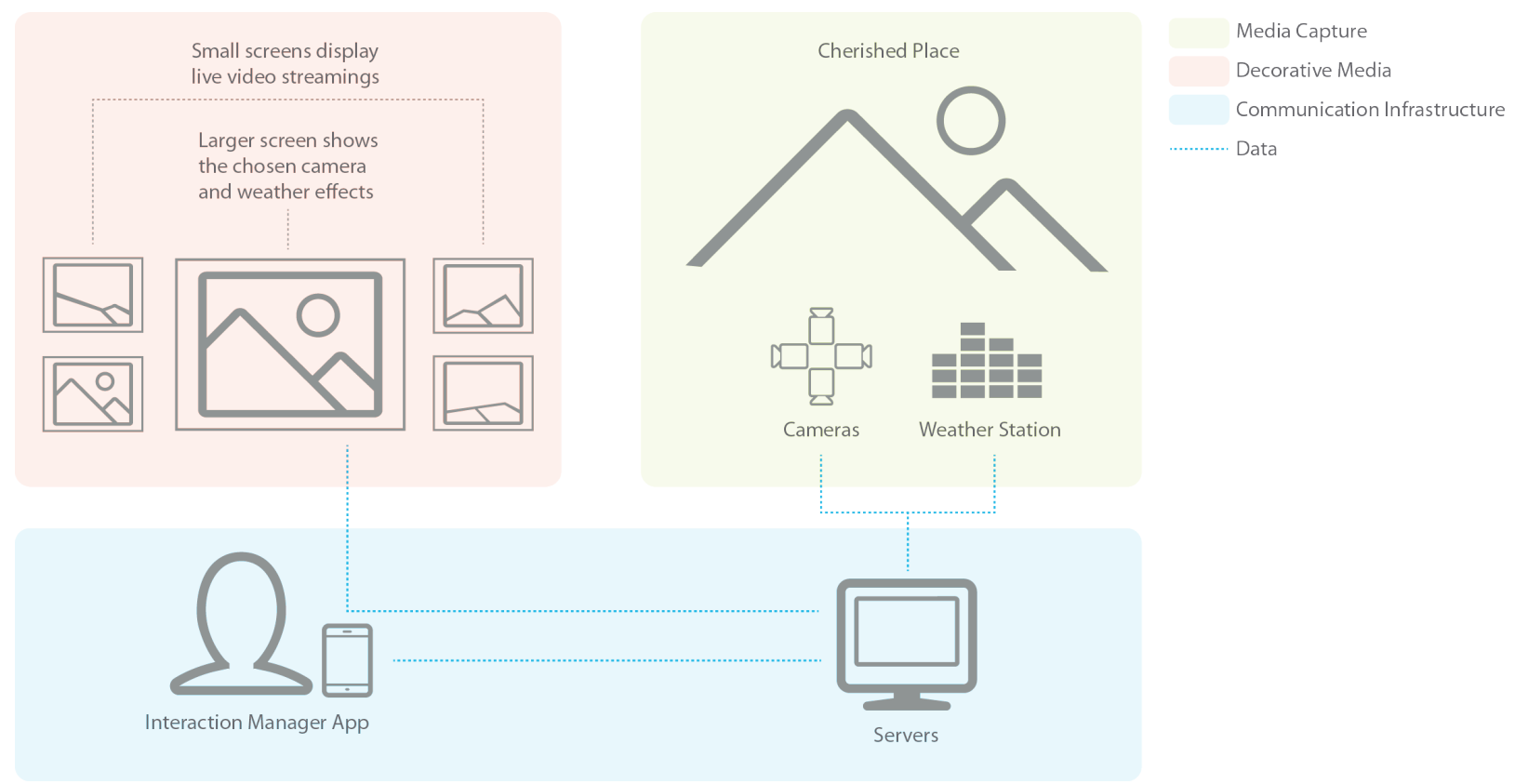

Figure 1: LiveNature design concept with media capture, communication infrastructure and decorative media.

looking out on the water...It's calm. It's tranquil." Third, a woman at the Island of Småskär said that she conceived of it when planning for the grocery shopping.

The interviews also revealed an understanding of when the cherished location was brought to the attention. In the case of relaxation it was considered during stressful parts of the daily work or just before falling asleep. The longing was triggered when a trip was approaching, or when actually seeing people travel to the cherished place, or when looking at snow in the woods and then thinking of Åre when walking the dog, or when looking at people skiing on TV.

In sum, their visualizations are of various kinds, and some of them include views of landscapes, sunlight and open seas, as well as people doing things. The images in the mind's eyes presented above are in no sense an overview of such daydreams. Still, we argue that they are enough to inspire us to design a system that catches aspects of those views in order to support an enriched remote experience of a cherished place.

\subsection{Design instantiation}

In this section we present the design concept including a brief overview of the process and our design intent.

During design process we used five keywords as the guidelines for the explorations i.e. ambience, liveness, individuality, hybridity and aesthetics. Several ideas were generated with three of them further developed to low-fidelity prototypes. These prototypes were installed in our office space and went through several pilot studies with visitors and research colleagues. One concept, i.e. the LiveNature system, was favored over the others and then further modified through several iterations. The concept was then conceived as three integrated components (Figure 1): a media capture device that record weather data and live video from the remote cherished place, a communication infrastructure including an interaction manager which enable the users to interact with the system, and decorative media in the home that display the live videos in their original form or mixed with weather data into a hybrid format.

\subsubsection{Media capture at remote cherished places}

We conceive of a set of mobile phones capturing live views. We intend to present the views of sky from the cherished place, and clouds in particular, as the subject of the live video streaming. It goes along with our idea of clouds as a trigger that encourages the user to dream about a cherished place in their mind's eye, rather than provide a visual "replica" of the place. We also intend to include other real-time sensor data such as those related to the weather e.g. temperature, wind speed and humidity and visualize it in combination with the video.

The system must have a communication mechanism, including servers that connect the remote place to the home. It is also in this system that generates real-time hybrid media i.e. where the data is translated into visual effects. We also conceive of an interface, called the interaction manager, where the users can manipulate the presentation.

The interaction manager (Figure 2) is the interface that enables the user to choose which camera feed to be displayed on the large screen and which sensor data that should influence the visualizations.

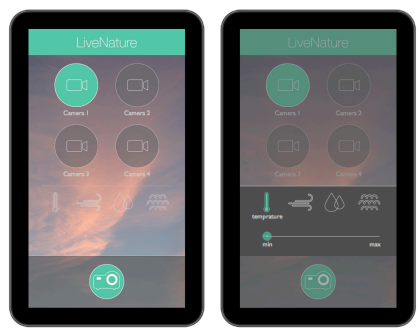

Figure 2: The interaction manager

\subsubsection{Decorative media in the home}

For aesthetical and decorative considerations, it is important to avoid the screens looking like computational components. Through a series of experimentations, the final design (Figure 3) include mobile phone and a large flat monitor all mounted in 
picture frames, distributed in different places in the home and blended in with other home decoration items. The four mobile phone screens display each camera view of the sky above the cherished place. The large screen displays a chosen camera view with the visual effects influenced by the real time weather sensor data from that place. To emphasize the decorative value and aesthetic experiences, the sensor data collected from the weather station is mapped to different visual effects that can be applied to the large screen. The design goal is to redesign a collection of mappings and enable the users to customize the ways of improvising that make sense to their individual feelings. The provisional mapping scheme is described below:

Temperature: The temperature data gathered from the weather station influences the RGB channel of the output video. The higher the temperature is, the warmer the color temperature is applied.

Humidity: The saturation level of the video output is influenced by the humidity of the cherished place. The more humid it is, the more saturated the video becomes.
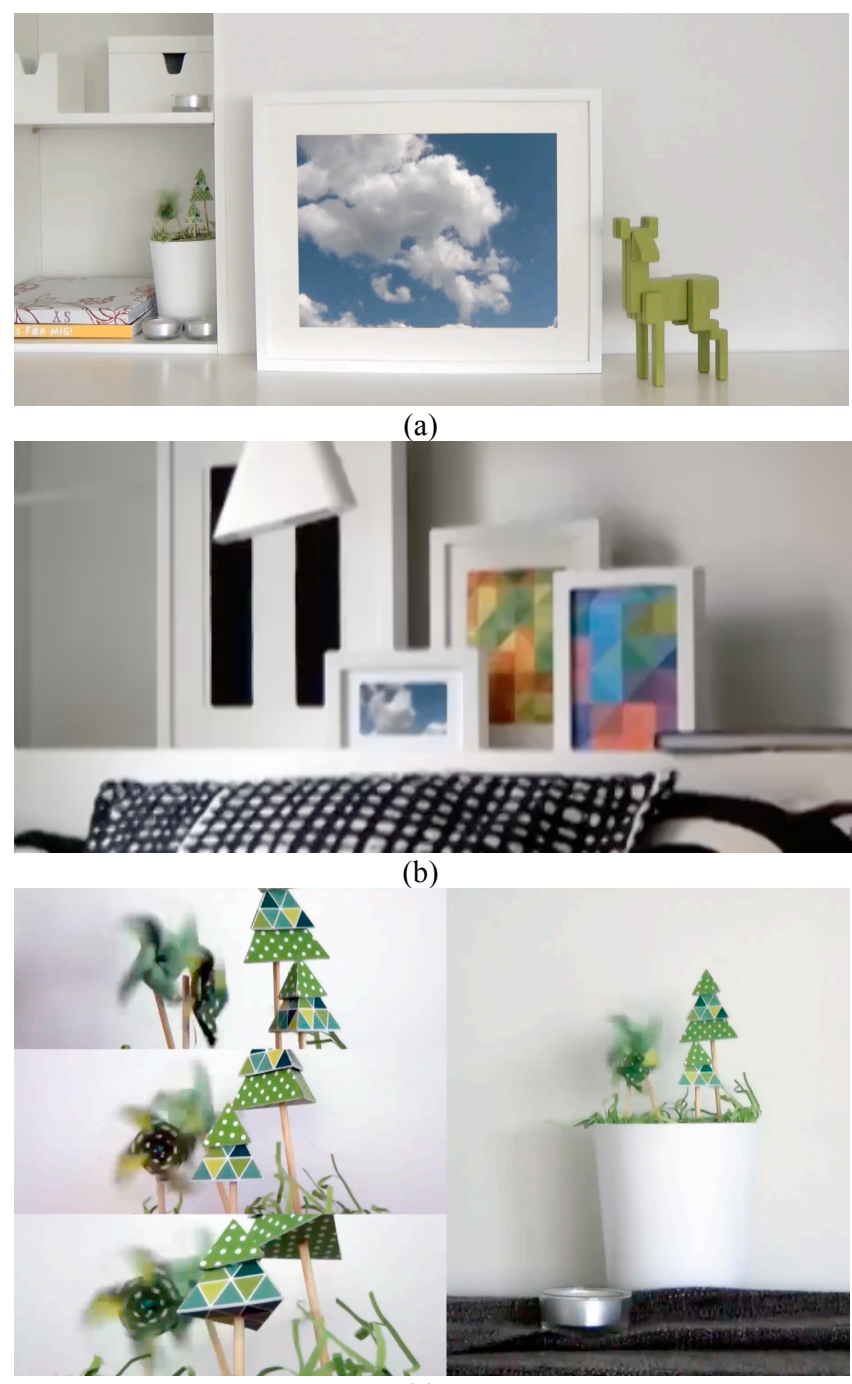

(c)

Figure 3: Large display (a), small screen (b) and windmill (c)

Air pressure and wind speed: The air pressure will change the contrast of the decorative video. The contrast will increase with increased pressure. The wind speed will change the degree of blur to the final video output dynamically. They higher the wind speed is, the blurrier the video becomes.

To expand the aesthetic interactions hybridity beyond the visualizations, we connected the "sense" of a remote place with the decorative item at home. The spinning motion of the windmills indirectly illustrates a sense of the cherished place and is intended to trigger an imagination of that place without presenting formal data (Figure 3c).

\subsection{Implementation}

Figure 4 illustrates the system architecture of the implemented prototype. We divide the system in three parts i.e. media capture, communication infrastructure and decorative media. The media capture part is responsible for seizing real-time video streams and real-time weather data. The communication infrastructure process and delivers data to the decorative media.

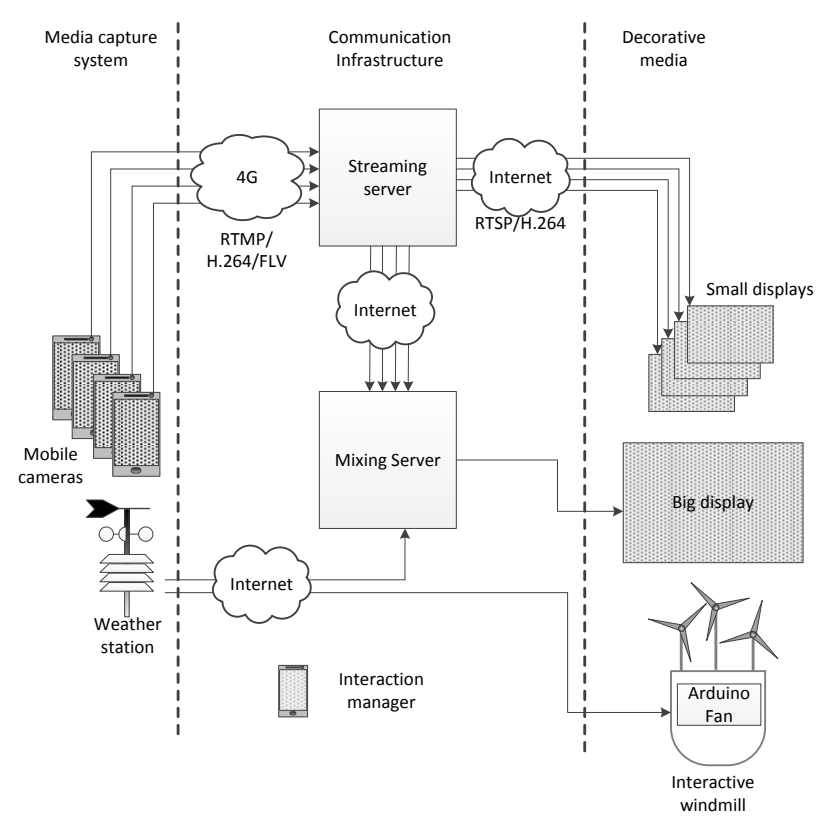

Figure 4: Detailed system architecture

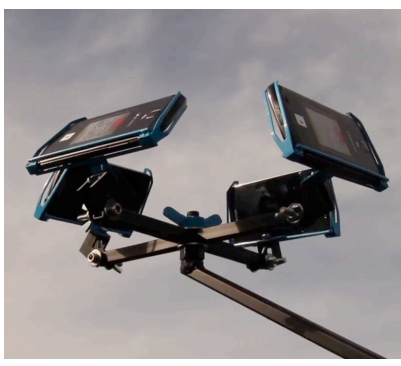

(a)

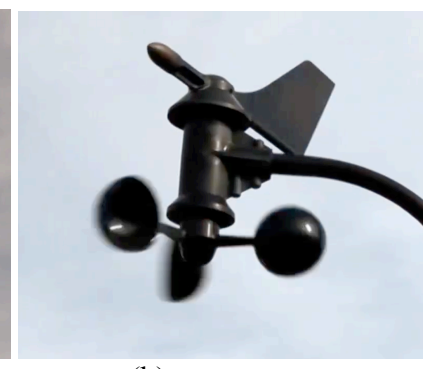

(b)
Figure 5: Custom built mobile phone stand \& Weather Station

The media capture system consists of four water proof mobile phones (Samsung Galaxy S4 Active which is IP67 rated for water and dust resistance), which are placed at a custom built "stand" attached to a pole (Figure 5 a). We made sure that all the power connections were waterproof by sealing them with plastic tape. The mobile phones stream live video to a streaming server using an FFmpeg based Android application that we developed. 
We also attached a weather station to the stand (Figure $5 \mathrm{~b}$ ), which measures temperature, humidity, air-pressure, wind-speed and wind direction.

The decorative media is implemented to display the captured video and the captured weather data in an aesthetic way. We used four Android mobile phones to act as small displays, referred to as the "small screens" that receive live RTSP streams from corresponding cameras. We fitted them into picture frames as shown in figure $3 \mathrm{~b}$. The live streams were received using a proprietary application called Vplayer due to its user friendly GUI.

The interactive windmill was implemented using a Yun Arduino board that controls a fan, which in turn, causes a hand-made paper ornament to rotate (Figure 3c), i.e. representing the wind speed at the capture location. The board is connected to the Internet through which it receives sensor data.

The communication inftrastructure consists of a mobile data network, a streaming server, a mixing server and an interaction manager. The live streams are transported to the streaming server using real time messaging protocol (RTMP) with H264 codec in FLV container over 4G/LTE network. RTMP is based on transport control protocol (TCP). We chose this method to ensure streaming over mobile networks (4G), since most operators do not allow user datagram protocol (UDP) based traffic. We use Wowza streaming server that is able to deliver streams in real-time using the RTSP protocol.

The mixing server is implemented in Max MSP/Jitter, which allows a variety of tools for multimedia design ${ }^{1}$. It receives video data from the streaming server via Internet and allows users (with the interaction manager) to select any of the four camera feeds to be displayed on the large display (Figure 3a) connected via the VGA/DVI port to the mixing server. It also fetches live weather data via Internet from the weather station. When a user selects to see weather information in the selected video feed on the large display, the mixing server applies appropriate visual effects corresponding to the selected weather parameter(s). For example if users selected temperature, the mixing server will automatically apply pre-defined visual effect on the selected camera feed in real time. The intensity of the visual effect corresponds to the value of temperature at the media capture location

The interaction manager is developed on Android, and it acts as a remote control that allows user to select among four camerafeeds as well as to enable/disable the hybrid visual effects. It communicates with the mixing server over Wi-Fi network.

\section{Technological challenges and evaluation}

The implemented system has to address a set of characteristics in order to provide for the suggested blend of mobile webcasting and ambient video. In the following we articulate these challenges and evaluate how the system performed.

\subsection{Challenges}

Long term continuous streaming: Generation of ambient video requires continuous streaming of both weather data and real time video for an extended period of time with minimal supervision and maintenance.

Video quality and multiplicity of streams: Home decoration and ambient video require high quality video, which must at least be

\footnotetext{
${ }^{1}$ http://cycling74.com/
}

equivalent to standard spatial definition $(640 * 480)$ at frame rate of $24 \mathrm{fps}$.

Data compression: The system must use an advanced and flexible (configurable) data compression mechanism for streaming to minimize the data transmission costs.

Mobility: The system should be mobile and utilize mobile Internet connections (e.g. 4G) for data streaming, since a cherished place can be located out-doors or away from the fixed Internet.

Hybridity: The system must be able to receive weather data from weather sensors and video streams from live feeds and mix them together in an aesthetical and meaningful way.

Glance-based and unobtrusive interaction: Ambient video requires glance-based interaction that does not interfere or disturb other activities at hand.

\subsection{Evaluation and lessons learned}

For technical evaluations we installed the decorative media part of the prototype in a test apartment and installed the media capture system on a balcony of an office building. We conducted a test of two weeks continuous deployment.

\subsubsection{Media capture system}

Long-term continuous operation: The system was running and streaming for 14 days to test the requirement of long term continuous streaming. The media capture system sustained 13 $\mathrm{mm}$ rain in the two weeks long period. It successfully streamed continuously and uninterrupted. We did not need to interrupt or supervise the media capture side during the period. Thus, the system fulfilled the stated requirement.

Video Quality: The system was able to support minimum standard definition resolution (640X480). We measured average frame rate on the receiving side by analyzing it using FFMPEG. The average frame rate was $14.6 \mathrm{fps}$. The frame rate received from the streaming application depends on many factors including processing power of the streaming hardware, processing load at the streaming/receiving hardware, and network bandwidth available.

Data compression: The streaming application that we developed for live streaming uses x 264 for encoding live stream. We tested encoded live video stream with average bitrates of $1500 \mathrm{Kbps}$, $1000 \mathrm{Kbps}$ and $700 \mathrm{Kbps}$ using profile baseline, level 3.0 with present ultrafast. We chose $1000 \mathrm{Kbps}$ given high requirements on quality. With this compression rate each mobile phone generated and streamed 10.2 Gigabytes of data per day.

Hybridity: The weather information is updated every 30 seconds and transmitted continuously over the period, which ensured that we could mix the live streams with other sensor data.

\subsubsection{Decorative media and Communication Infrastructure}

Long-term continuous operation: Due to the ambient nature of project the screens are supposed to receive and display the video streams continuously 24 hours a day. We kept the small screens connected to the chargers so they do not go out of battery.

The evaluation showed that these mobile devices were turning off several times. When a device was shut off it was necessary to manually turn them on and launch the Vplayer application again. On investigating we found that they were not charging properly since we were using extended USB charging cables. The added length of the USB cable affected the charging rate of 
the phones. The extended cables were required by the furniture manufacturer's stakeholder since the charger was seen as unaesthetic and had to be concealed. Replacing the cables with the shorter ones solved the charging problem.

The mixing server was also turned off at least twice due to the power failure and the server application crashed three times during the period of two weeks.

High-resolution displays and glance-based interaction: Most smart phones come with high-resolution displays. The challenge is then how to locate them in a way that provides both glance based and unobtrusive interaction. We chose to position the large display (Figure 3a) at the end of a hallway near the kitchen's door, which was also close to the shared bathroom. It is likely that users will pass by it and have a glance at the sky (mediated by the large screen). We installed the rest of the components in decorative media on places where people normally place their decorative objects to encourage the glancebased interaction. We did so because such places are not likely to be the focus of attention in a normal household setting.

Hybridity: The mixing server was able to produce real time video visualization from sensor data and mix these visualizations with the selected live video stream. The fan, i.e. the interactive windmill, was spinning and the wind data was received as expected only occasionally. There were several occasions when the fan stopped working, thus showing instability.

Unobtrusiveness and ease of use: The requirement of unobtrusiveness on the decorative media proved to be challenging.

The interactive windmill (Figure 3c) produced a noise from its mechanics, which was rather unpleasant. The mixing server, with its cooling fan, is co-located with the large display was also producing noise that was noticeable during nights when other sounds were at the minimum.

The interaction manager was able to control the mixing server's functionality with an average delay of 300 milliseconds. This delay is due to the server's internal implementation and processing and propagation delay over Wi-Fi. While testing the interaction manager we found that sometimes the command is not transmitted to the server.

In sum, we learned that most of the system features worked to our satisfaction and lived up to the requirements. It was difficult to achieve uninterrupted streaming and we need more investigation and improvement in the mixing server application to avoid random crashes. It is also important to provide uninterrupted power supply, while meeting the requirements of home décor. The same goes for the sound levels where the noise has to be minimized to provide unobtrusive interaction.

\section{DISCUSSION}

This paper presents a design investigation that the potential of a system concept, which combines the mobile webcasting technology with the ambient video format for domestic environments. It was conducted by generating a concrete design instance, influenced by ethnographic field studies. We also combined emerging technology, such as mobile smart phones and high definition displays, with custom built hardware and software to cope with the practical design challenges. The design instance serves as an exemplar to indicate and articulate the potential of combining technologies to realize a novel live video format. The result contributes to the understanding of the use of this emerging technology for enjoyment in the following sense:

\subsection{Mobile webcasting as extension to ambient video}

The design research provides a better understanding on the potential of extending ambient video with mobile webcasting technology.

First, it is stated that in ambient video the broadcast should be continued which then requires that the mobile system provide continuous streams. The two-week experiment where four video feeds were broadcasted without interruption is promising, and an indication that liveness from personalized setting is an emerging option to extend the artistic ambient video format. Our evaluation shows that we need to encode h264 live video stream with average bitrate of $1000 \mathrm{kbps}$ to meet quality requirements, which generate as much as 70 Gigabyte per week per mobile camera. Since we used free mobile data subscription from an industrial partner, we were not charged with any costs. Even though it has not been targeted in this research project, it is obvious that a future deployment of LiveNature would be expensive with most available price models for mobile Internet, and it is necessary to account for these aspects.

Second, ambient video is considered to be presented in high resolution, which obviously depends on high quality video streams. Mobile webcasting is still far from providing the video quality that can be achieved in wired networks, but the recent shift from $3 \mathrm{G}$ to $4 \mathrm{G}$ networks has made a difference. In our case we were able to provide video streams in the standard definition $(640 \times 480)$ at 14.6 fps. Since we focus on slowly moving natural sceneries, as part of investigating the ambient video format, we are able to compromise with the amount of frames per second on behalf of the screen resolution. The result shows good quality on the output screens, except when birds rapidly pass over the cameras. The system performed in specific settings during our test period. Since the system's performance depends on several factors (e.g. temperature, network condition etc.) therefore the system can behave differently in varied settings.

Third, ambient video intends to display slowly altering content, such as natural sceneries. Previous such videos have been preproduced by selected artists for other people to view. LiveNature adds to the format by providing liveness and personalization since inexpensive rugged off-the-shelf mobile devices can be located in individually selected natural environments.

Fourth, it is argued that viewers' interaction with ambient video should be casual and only occasionally focused. Mobile webcasting allows individualized content, which then needs to be presented in some personal context such as in their homes. We therefore choose to design screens and windmill to fit with other home decoration, and allow interaction with LiveNature in the same way as we interact with decorative objects such as paintings on the walls and figurines standing on shelves. Our design solutions convinced the large furniture manufacturers research department of including the system in a larger test apartment were families would live on a bi-weekly basis, and explore a set of new products.

In sum, mobile webcasting is an emergent technology field that could bring liveness, personalization and hybridity into ambient video. That opens for a broader use context for this video format, beyond that of the art scene. 


\subsection{Ambient video as new format for mobile webcasting}

The research provides a better understanding on the potential of sustaining and developing mobile webcasting with the ambient video format. It would then extend the two previously referred formats of "production" webcasting and "snapshot" webcasting. Users of mobile webcasting struggle with the amount of production work required and finding stories to tell.

First, ambient video has to require low effort of the producer. The idea with LiveNature is that the broadcast is automatic and will continue over months or years. The work involved consists of installing the system at a cherished place. Our designed stand (figure 5a) ensures that it can be done with ease. Based on our own experience, the phones are quickly attached and directed in a favored direction. With the cameras and the weather station in place, the user is also required to administer the broadcast through the interaction manager (figure 2).

Second, users of mobile webcasting testify that they struggle with finding relevant broadcast content. The design of the LiveNature system draws on our study of people who have a strong relation to a cherished place. We suggest that having live access to such a place would provide meaningful content and a story to tell i.e. the weather at that location, which in turn would evoke reveries of days passed and days to come.

In sum, ambient video in this version has particular features, such as the consistent streaming and aesthetic content, which make it a third and complementary option to mobile webcasting. The research indicates that the format could emerge as an alternative among others in the investigation of the live video design space.

\section{Conclusion}

Future use of mobile webcasting technology is uncertain. The production of live video by amateurs who work with professional standards is rather limited. Recently we have seen an emerging turn to snapshot photography as model for video as social media. The development indicates that this medium is still in its infancy and suggests that even more formats will emerge. In this paper we suggest that new models and formats in general, and that of ambient video in specific, might spark future use of the affordances of this emergent technology. The design instantiation presented in this paper and its technical evaluation are promising in the sense that LiveNature provided continuous streaming of moderate quality video to be displayed and utilized for home décor. The study also initiates a request for a more elaborated theoretical understanding of the use of aesthetics in home decoration, as well as a better understanding of mundane user demands on ambient video services in the domestic domain. Finally, it remains to be studied if the system provides interesting experiences beyond the technical characteristics investigated here. So, the next step would be to conduct a user study to investigate the experience offered by the proposed system.

\section{REFERENCES}

[1] Al-Ani, B., Mark, G., Chung, J. et al, The Egyptian blogosphere: a counter-narrative of the revolution. In Proc. CSCW '12. ACM, NY, USA, 17-26

[2] Anderson, A. "The 'New Old School': Furnishing with Antiques in the Modern Interior-Frederic, Lord Leighton's
Studio-House and Its Collections." Journal of Design History 24.4 (2011): 315-338

[3] Anind, K., et al. From awareness to connectedness: the design and deployment of presence displays. In Proc.CHI '06. ACM,

[4] Bizzocchi, J. 2003. The magic window: the emergent aesthetics of high-resolution large-scale video display. In Proc.ICEC '03

[5] Bizzocchi, J. 2005. Streaming video: an experiment in new aesthetics. In Proc.C\&C '05

[6] Bizzocchi, J. Ambient video. In Proc.ACE '06

[7] Bizzocchi, J. Winterscape and ambient video: an intermedia border zone. In Proc. MM '08

[8] Csikszentmihalyi, M. and Rochberg-Halton, E. The Meaning of Things : Domestic Symbols and the Self . P179 Cambridge University Press, (1981).

[9] Eno, B. Music for Airports, PVC 7908 (AMB 001) album liner notes, 1978

[10] Fogarty, J. Jodi, F. and Scott E. H. "Aesthetic information collages: generating decorative displays that contain information." In Proc. UIST' 01. ACM, 2001.

[11] Frayling, C. Research in art and design. Royal College of Art London, 1993.

[12] Gaver, W. 2002. Provocative Awareness. Comput. Supported Coop. Work 11, 3, 2002, 475-493.

[13] Gaver, W. and Bowers, J. Annotated portfolios. Interactions 19, 4 (2012), 40.

[14] Gaver, W. What should we expect from research through design? In Proc. CHI'12,(2012),937

[15] Gaver, W., Bowers, J., and Boucher, A. The drift table: designing for ludic engagement. In Proc. CHI'04, (2004), $885-900$

[16] Gaver, W., Bowers, J., Boucher, A., Law, A., Pennington, S., and Villar, N. The history tablecloth: illuminating domestic activity. In Proc. DIS'06, ACM (2006), 199-208.

[17] Gillmor, D. (2010), Mediactive. Published under a Creative Commons A-NC-SA license.

[18] Higgins, D., "Intermedia", 1965 and 1981, reprinted in Leonardo, Vol. 34, No. 1. (2001)

[19] http://blogs.skype.com/2012/08/28/the-advent-of-ambientvideo/ (Last accessed on $21^{\text {st }}$ May 2014)

[20] Juhlin, O., Engström, A., and Reponen, E.: Mobile Broadcasting: The Whats and Hows of Live Video as a Social Medium. In Proc. MobileHCI 2010, 35-43 (2010)

[21] Juhlin, O., Zoric, G., Engström, A., Reponen, E. (2013) Video interaction: a research agenda. J Personal and Ubiquitous Computing, Springer London, p 1-8 22.

[22] Kirk, D., Sellen, A. On Human Remains: Values and Practice in the Home Archiving of Cherished Objects. ACM Trans. Comput.-Hum. Interact. 17, 3 (2010), 10:1$10: 43$.

[23] Kirk, D., Sellen, A., Harper, R. \& Wood, K. 2007. Understanding Videowork. In proc. CHI'07, San Jose, USA

[24] Klemmer, R.S., Thomsen, M., Phelps-Goodman, E., Lee, R. and Landay, J.A. Where do web sites come from? 
Capturing and interacting with design history.

In Proc. CHI'02, ACM Press (2002), 1-8.

[25] Lehmuskallio, A., Sarvas, R. Snapshot video: everyday photographers taking short video-clips. In Proc. NordiCHI '08. ACM, NY, USA, 257-265

[26] Manovich, L. Understanding Hybrid Media. in Betti-Sue Hertz, ed., Animated Paintings San Diego: San Diego Museum of Art, 2007

[27] Mather, B.D. Making up titles for conference papers. Ext. Abstracts CHI 2000, ACM Press (2000), 1-2.

[28] Meese, R., Ali, S., Thorne, E.-C., et al. From Codes to Patterns: Designing Interactive Decoration for Tableware. In Proc. CHI'13, ACM (2013), 931-940.

[29] Miller, D. Stuff. Wiley, 2013.

[30] Miller, D. The Comfort of Things. Wiley, 2008.

[31] Sas, Corina, et al. "Generating implications for design through design research." In Proc. CHI'14. ACM, 2014.

[32] Scannell, Paddy. 1996. "Radio, Television, and Modern Life: a phenomenological approach."

[33] Schwartz, M. Guidelines for Bias-Free Writing. Indiana University Press, Bloomington, IN, USA, 1995.

[34] Sellen, A. Family Archiving in the Digital Age. In R. Harper, ed., The Connected Home: The Future of Domestic Life. Springer London, 2011, 203-236.
[35] Shirky, C.: Here comes everybody: the power of organizing without organizations. Penguin Books, New York (2008)

[36] Vihavainen, S., Mate S., et al. 2012. Video as memorabilia: user needs for collaborative automatic mobile video production. In Proc. CHI '12. 651-654

[37] Wadley, G. et al. (2013). Mobile ambient presence. In Proc. OzCHI '13

[38] Wang, T. et al. Video stylization for digital ambient displays of home movies. In Proc. NPAR '10.

[39] Ylirisku, S., Lindley, S., Jacucci, G., et al. Designing Webconnected Physical Artefacts for the 'Aesthetic' of the Home. In Proc. CHI'13, ACM (2013), 909-918.

[40] Youssef, B.B. et al. The future of video: user experience in a large-scale, high-definition video display environment. In Proc. ACE '05.

[41] Zellweger, P.T., Bouvin, N.O., Jehøj, H., and Mackinlay, J.D. Fluid Annotations in an Open World. Proc. Hypertext 2001, ACM Press (2001), 9-18.

[42] Zimmerman, J., Forlizzi, J., and Evenson, S. Research through design as a method for interaction design research in HCI. In Proc. CHI'07, (2007), 493-502.

\section{Columns on Last Page Should Be Made As Close As Possible to Equal Length}

